Service social

\title{
20 questions pour penser le travail social, de Jacques Riffault, Paris, Dunod, 2007, 199 pages
}

\section{Normand Brodeur}

Volume 55, numéro 1, 2009

URI : https://id.erudit.org/iderudit/029492ar

DOI : https://doi.org/10.7202/029492ar

Aller au sommaire du numéro

Éditeur(s)

École de service social de l'Université Laval

ISSN

1708-1734 (numérique)

Découvrir la revue

Citer ce compte rendu

Brodeur, N. (2009). Compte rendu de [20 questions pour penser le travail social, de Jacques Riffault, Paris, Dunod, 2007, 199 pages]. Service social, 55(1),

111-115. https://doi.org/10.7202/029492ar d'utilisation que vous pouvez consulter en ligne.

https://apropos.erudit.org/fr/usagers/politique-dutilisation/ 


\title{
Compte rendu
}

\author{
Jacques RIFFAULT, 20 questions pour penser le travail social \\ Paris, Dunod \\ 2007, 199 pages
}

Jacques Riffault est éducateur et directeur adjoint de l'Institut régional du travail social d'Île-deFrance Montrouge/Neuilly-sur-Marne. Le recueil 20 questions pour penser le travail social est constitué d'articles qu'il a publiés, d'entrevues qu'il a accordées et de conférences qu'il a prononcées entre 1996 et 2006. L’ouvrage vise à répondre à la demande de réflexion philosophique qui émerge chez les travailleurs sociaux français, dans le contexte d'un champ qui cherche à se définir comme discipline à part entière, de la multiplicité des références - et donc de la relative fragilité des assises théoriques et conceptuelles - et des difficultés liées à la définition de son identité professionnelle. Il ne prétend pas ajouter de références supplémentaires à la boîte à outils des travailleurs sociaux, mais plutôt les aider à penser par eux-mêmes, à réfléchir aux questions relatives, aux fondements de leur activité, à la validité des savoirs qui leur sont disponibles pour la mener à bien et à ses finalités. Le livre est divisé en trois grandes sections qui abordent respectivement les valeurs, la connaissance et le sens des pratiques sociales.

Riffault a regroupé sous le thème des valeurs sept chapitres traitant de questions diverses. Le premier aborde les dimensions fondatrices de l'engagement professionnel en travail social. On y trouve non seulement la réaffirmation d'un certain nombre de valeurs telles que le refus des injustices, le respect de la dignité de la personne et la croyance en ses capacités et en celles de la société, mais aussi un appel à la rationalité. Selon l’auteur, celle-ci n’est « jamais donnée, mais toujours à construire » (p. 13). Elle renvoie notamment à la responsabilité des intervenants de rendre compte de leurs pratiques.

Le second chapitre présente les différentes formes d’intervention sociale comme des pratiques d'influence. Cette notion, apparentée à une capacité générale de persuader, suppose un consensus entre la personne qui influence et celle qui est influencée sur les finalités qui donnent sens à leur relation. Mais elle laisse aussi le champ ouvert à des analyses mettant en évidence les rapports de force plus ou moins implicites entre les protagonistes. Puisque l'influence repose sur une combinaison de légitimité institutionnelle, de compétences et de normes, l'auteur estime que l'éthique et l'épistémologie sont des éléments d’interrogation nécessaires et indissociables de la pratique sociale.

Le troisième chapitre porte sur la formation des professionnels. S’inspirant d'une expérience de formation sur la santé mentale et la lutte contre les exclusions auprès d'intervenants provenant de plusieurs horizons, l'auteur propose une formation interprofessionnelle permettant aux professionnels « de sortir de leur spécialité et de faire autre chose que ce pour quoi ils ont été

Service social - Volume 55, numéro 1, 2009 
formés initialement, sans pour autant perdre leur identité »(p. 39). Pour que cet objectif soit atteint, écrit-il, la formation initiale doit miser sur le développement d'une culture philosophique et sur l'écriture, de façon à ce que les futurs intervenants adoptent une position de praticien chercheur.

Le quatrième chapitre explore un thème devenu tabou à la suite des travaux de Michel Foucault, soit celui de la sanction. Riffault soutient que l'éducation ne peut se passer de la contrainte, tout en précisant les conditions sous lesquelles l'éducateur peut légitimement en faire usage. Ainsi, le projet de l'éducation étant « la constitution de l'humanité dans l'homme [sic] » (p. 47), le recours à la sanction ne trouve sa légitimité dans la relation éducative que lorsqu'il permet d'ouvrir l'espace de l'échange. Il nécessite de plus un travail intérieur constant permettant à l'acteur de reconnaître la possibilité de céder lui-même à la violence et de se dérober à sa propre obligation d'échanger.

Le cinquième chapitre tente de définir la praxis. Celle-ci se rapporte au fait même d'agir. Elle désigne "cette modalité de l'action qui ne se laisse pas réduire à l'application d'un plan prédéterminé, qui a pour objet “le non advenu”, l'imprévu, marque de l'humain créateur de réalité et de sens nouveaux, qui, transformant son monde, se laisse aussi transformer lui-même au cours de cette activité » (p. 58-59). L'auteur y voit une notion très utile pour les éducateurs dont le projet est de permettre le développement et l'actualisation des possibilités dans le contexte d'imprévisibilité qui découle nécessairement de l'affirmation de la liberté humaine.

La section se termine par deux courts chapitres dans lesquels l'auteur fait valoir d'une part, la pertinence de la psychanalyse en tant qu'axe structurant pour la pensée des travailleurs sociaux et, d'autre part, l'importance du travail comme pièce maîtresse des processus éducatifs par lesquels les êtres humains deviennent ce qu'ils sont et accèdent à leur humanité. Ce dernier élément vise particulièrement à mettre en valeur la contribution au travail social des éducateurs techniques dont la responsabilité consiste à enseigner un métier aux adolescents en difficulté.

La section portant sur la connaissance comprend sept chapitres et est la plus volumineuse de l'ouvrage. Son contenu est toutefois plus homogène que celui de la section précédente. Deux thèmes majeurs y sont traités, soit celui du statut des références théoriques en travail social et celui de l'écriture professionnelle.

Dans un chapitre portant sur les préalables à l'étude des processus de référenciation théorique en travail social, Riffault estime que les principaux outils du travail social sont la parole, le désir et la relation. Selon lui, les théories n'en demeurent pas moins nécessaires, pour peu qu'on cesse de les voir comme un réservoir d'approches ou d'outils pour la pratique. Le rapport entre la théorie et la pratique n'en est pas un d'application, dans lequel la première commanderait à la seconde, mais bien un rapport d'investissement intellectuel et culturel ayant pour effet l'élargissement de la conscience et du pouvoir d'action. En effet, la production de théories étant une manière de se rapporter au monde qui consiste à essayer de le connaître et de l'interpréter pour qu'il devienne 
provisoirement sensé et ordonné, les références théoriques constituent une matière qui participe à la construction personnelle des "sites" sur lesquels les travailleurs sociaux intériorisent et élaborent en eux-mêmes un support pour leur conduite. Plus ces sites sont riches et nombreux, plus leur adaptabilité est grande et plus leur travail clinique peut être fin et rigoureux. Ces idées sont approfondies davantage dans le chapitre suivant dans lequel l'auteur examine le chemin de formation qui permet au sujet professionnel d'advenir dans la relation aux références qui donnent un caractère rationnel à son travail. En parcourant ce chemin, les travailleurs sociaux doivent progressivement cesser de voir la référence théorique comme une idéologie totalisante et la considérer plutôt comme un investissement parmi d'autres leur permettant de développer des réponses possibles aux problèmes qu'ils rencontrent. Pour favoriser ce travail de désintrication et contribuer à l'avenir du professionnel en tant que sujet, le rôle des formateurs est de confronter le futur professionnel à la nécessité des références théoriques tout en lui permettant de s’interroger sur leurs fondements.

Riffault consacre trois chapitres à l'écriture professionnelle, tous basés sur des enquêtes auprès d'intervenants sociaux. L'auteur évoque divers malaises liés à l'écriture comme, par exemple, la difficulté d'identifier ce que l'on peut dire dans un rapport sans que cela n'intervienne trop dans l'intimité d'une personne, la piètre qualité des écrits qui suscitent la méfiance chez certains collaborateurs des travailleurs sociaux ou encore l'impression, chez ces derniers, que l'écriture constitue une tâche administrative supplémentaire déconnectée de leur pratique. La cause de ces malaises se trouverait dans les modèles de communication proposés aux travailleurs sociaux qui s’inscrivent dans une épistémologie positiviste obsolète. Dénonçant le mythe selon lequel ils pourraient être des observateurs objectifs et impartiaux des situations dont ils ont à rendre compte, alors que celles-ci sont en réalité coconstruites par eux, l'auteur propose de réhabiliter la subjectivité en tant que source de toute relation, de toute pensée, de toute connaissance et de toute écriture. À l’idée d’objectivité, il substitue celle de processus d’objectivation dans lequel la réflexivité occupe une place centrale. Cette nouvelle épistémologie donne un nouveau sens à l'écriture professionnelle, car elle est un lieu privilégié de remise en question des images que l'on se fait des personnes et des situations. Cette écriture subjective doit être argumentée et discutée avec les autres acteurs, y compris les personnes concernées par l’intervention, jusqu’à ce qu'une représentation commune émerge.

La dernière section de l'ouvrage interroge le sens de certaines pratiques sociales et plus particulièrement celui du travail auprès des personnes présentant de graves incapacités. Dans les quatre chapitres sur six consacrés à ce thème, Riffault aborde trois sujets principaux. Le premier est celui de la maltraitance et de la violence envers les personnes handicapées. Selon la perspective psychanalytique adoptée par l'auteur, ce phénomène serait lié non seulement à la disproportion entre les forces des uns et les faiblesses des autres, mais aussi, et surtout à la violence initiale et fondatrice du "handicap" lui-même qui induit, chez les parents comme chez les soignants, une blessure narcissique, un sentiment de culpabilité dont ils cherchent à se défendre. Dans ce contexte, la réflexion et l'interrogation constante des souffrances vécues par les personnes handicapées, leur famille et les personnes qui travaillent auprès d'elles 
constitueraient le principal antidote aux mauvais traitements. Le second sujet est celui de l'expression de la sexualité chez les personnes handicapées et de la peur qu'elle suscite. Dans le cas de la déficience intellectuelle, par exemple, la possibilité que l'enfant puisse à son tour donner la vie évoque la possibilité du retour de la souffrance initiale vécue par les parents. Ceuxci sont alors portés à se demander par quels moyens les effets dévastateurs de l'éveil de cette sexualité peuvent être limités. Or, cette question s'inscrit dans une logique utilitaire que l'auteur cherche à déconstruire. Car la sexualité va pour lui au-delà du comportement sexuel. Elle est l'expression du désir de vivre, d'une aspiration au plaisir et au bonheur. Elle est source de création. Dans une perspective qui unit l'âme et le corps plutôt que de les séparer, la vie affective et sexuelle des personnes présentant une limitation intellectuelle devient une expérience possible. Elle peut être conçue comme une question relevant de la vie pratique et d'un art de vivre plutôt qu'un problème à traiter. Finalement, le dernier sujet est celui de l'habitation en contexte de handicap. Du point de vue de l'anthropologie philosophique, l'habitat est un chez soi, une matérialisation de l'espace psychique de la personne. Il a pour fonction de permettre à une subjectivité de faire l'expérience d'elle-même. Plutôt que d'être étudiées uniquement sous l'angle restrictif du logement et des difficultés de la vie matérielle, les questions relatives à l'habitation des personnes handicapées doivent donc être pensées de façon à " permettre à une subjectivité de s'habiter, de se sentir chez elle dans ce qui est, dans ce qu'elle est, et parmi les autres... pour mieux habiter le monde et ainsi en être moins exclue » (p. 191).

20 questions pour penser le travail social offre une réflexion riche et dense sur les enjeux de l'intervention sociale contemporaine. Que l'on partage ou non les thèses soutenues par l'auteur, il faut reconnaître le courage et la rigueur dont il fait preuve dans le traitement de questions controversées et délicates comme la sanction, l'autorité, la violence envers les personnes handicapées ou l'expression de la sexualité chez ces dernières. L'ouvrage s’avère aussi original dans le choix de certains thèmes, dont celui de l'écriture professionnelle. En effet, alors que la rédaction de dossiers et de rapports constitue aujourd'hui une activité centrale pour la plupart des travailleurs sociaux, il existe à ma connaissance peu d'études et d'analyses à ce sujet. Les étudiants, les praticiens et les gestionnaires ne trouveront pas dans ce livre un guide sur la tenue des dossiers, même si l'auteur esquisse quelques options autres que la traditionnelle description « objective » des faits. Ils trouveront toutefois matière à remettre en question les fondements épistémologiques de cette pratique et à la renouveler.

Le point de vue de l'auteur sur la place et le statut des théories dans la pratique du travail social et dans la formation n'est pas entièrement nouveau. À l'instar de Riffault, qui y voit « une régression intellectuelle, sociale et politique » (p. 110), bien d'autres commentateurs ont dénoncé l'emprise du positivisme et de la rationalité instrumentale dans les sciences humaines et sociales. Les chapitres qui y sont dévolus ne sont pas pour autant dénués d’intérêt. Ils synthétisent habilement les principales thèses relatives à l'articulation théorie/pratique sous l'angle du constructivisme. À l'heure des meilleures pratiques et des interventions basées sur les données probantes, cette critique de l'épistémologie dominante demeure d’actualité. 
Comme je l'ai souligné en introduction, ce livre a d’abord été conçu pour un public français. Les travailleurs sociaux québécois auront peut-être quelques difficultés à se reconnaître dans les exemples proposés dans le livre. Ainsi, une description plus exhaustive des « actions éducatives en milieu ouvert - AEMO », à laquelle l'auteur se réfère dans quelques chapitres, aurait été souhaitable. Plus fondamentalement, la notion même de «travail social » couvre dans cet ouvrage un champ disciplinaire plus large que celui auquel on se réfère habituellement dans le système professionnel du Québec. Les intervenants auxquels l'auteur s'adresse exercent en fait plusieurs professions jouissant ici d'une identité distincte, tels que les travailleurs sociaux, les psychologues et les éducateurs spécialisés. De ce fait, d’aucuns s'étonneront de la place accordée à l'éducation dans cet ouvrage dédié au travail social, alors que cette tâche, au Québec, est généralement confiée à une autre catégorie de professionnels. Cet obstacle sera toutefois surmonté, pour peu que l'on sache voir ce que les métiers du social ont en commun plutôt que de se concentrer que sur leurs particularités.

Normand Brodeur

Professeur adjoint

École de service social

Université Laval 